The information in this column is not intended as a definitive treatment strategy but as a suggested approach for clinicians treating patients with similar histories. Individual cases may vary and should be evaluated carefully before treatment is provided. The patient described in this column is a composite with characteristics of several real patients.

\section{Inappropriate benzodiazepine use in elderly patients and its reduction}

\section{Cara Tannenbaum, MD, MSc}

A 72-year old woman with a psychiatric history of anxious depression and insomnia is receiving ongoing psychotherapy and psychotropic management. Her mood disorder has been stable with escitalopram $(10 \mathrm{mg} / \mathrm{d})$ for 3 years. She has been taking lorazepam (1 mg nightly) since the death of her husband 12 years ago. Recent research about chronic benzodiazepine therapy leading to an increased risk of Alzheimer disease prompts a discussion about benzodiazepine cessation. ${ }^{1}$

Benzodiazepines and other types of sedative-hypnotics, such as Z-drugs, are no longer recommended for treating insomnia in older adults and are considered inappropriate. ${ }^{2}$ In addition to causing memory impairment, falls, fractures and motor vehicle accidents, ${ }^{3-6}$ data now show that sedativehypnotics account for a substantial number of avoidable emergency department visits and hospital admissions. ${ }^{7}$ Even episodic use is associated with harm. A lifetime use of more than 90 doses of benzodiazepines, equivalent to twice a week for 1 year, has been shown to confer a $50 \%$ higher risk of dementia and to double the risk of death. ${ }^{1,8}$ The risk of hip fracture is greatest within the first 2 weeks of therapy, increasing with higher doses and concomitant administration of other centrally acting nervous system drugs. ${ }^{9}$

Patients may not be aware of the risks of chronic benzodiazepine use. ${ }^{10}$ Informing them of the latest research findings may elicit a desire to taper. Level 1 evidence supports patient education as an effective method for catalyzing benzodiazepine reduction. ${ }^{10}$ The EMPOWER trial exposed
150 chronic benzodiazepine consumers aged 65-95 years to a mailed 8-page educational brochure (www .criugm.qc.ca/images/stories/les _chercheurs/risk_ct.pdf) on the risks of taking sedative-hypnotics, along with a picture of a 20-week tapering protocol, showing when to take a full-, half- or quarter-pill dose to gradually withdraw from therapy. ${ }^{10}$ Within 6 months, $27 \%$ of individuals who received the intervention had completely discontinued use compared with $5 \%$ of controls, and an additional $11 \%$ had reduced their dose. ${ }^{10}$ Another randomized trial ${ }^{11}$ in primary care showed that distribution of a written tapering protocol along with a 20-minute physician-patient discussion about benzodiazepine cessation led to a $45 \%$ reduction in use at 1 year follow-up, even without close monitoring. Patients with severe psychiatric disorders on antipsychotic therapy were not included in either trial.

No magic formula exists for tapering benzodiazepines, as different protocols have not been compared. Some authorities recommend tapering the dose by $25 \%$ every 2 weeks; in elderly patients a longer tapering schedule over $4-5$ months is generally preferred. ${ }^{10-13}$ Withdrawal symptoms tend to be most severe during the last quarter of the taper. ${ }^{13}$ Updosing (returning to a higher dose) should be avoided. Patients should be maintained on their current doses until symptoms resolve or be encouraged to push through the taper until they are drug-free. ${ }^{13}$ Substitution with diazepam was previously recommended for formulations of benzodiazepines that could not be halved or quartered, but skipping doses every $2-3$ days is a simpler strategy to gradually reduce drug levels.

Patients and providers hesitate to discontinue benzodiazepines because of fear of withdrawal symptoms or relapse. ${ }^{10,14}$ Withdrawal symptoms occur in up to $50 \%$ of patients who succeed in tapering. ${ }^{11}$ Symptoms of insomnia, tremor, irritability and anxiety are usually transient, and at 1-year follow-up they are no different in frequency between patients who do not taper and those who do. ${ }^{11}$ Perceptual disturbances, gastrointestinal symptoms and seizures rarely occur. ${ }^{11-13}$ No serious safety events were reported in a systematic review of 28 studies of benzodiazepine tapering among older adults with insomnia, depression and anxiety. ${ }^{11-13}$

Cognitive behavioural therapy is effective for treating chronic insomnia and facilitating benzodiazepine tapering in older adults. ${ }^{15-17}$ Maintaining good sleep hygiene and using a sleep diary to monitor sleep efficiency during or after benzodiazepine withdrawal can be helpful. ${ }^{12,15-17}$ Patients are often reassured by the knowledge that normal sleep architecture changes with age and that older adults can be expected to sleep for fewer hours each night and experience more awakenings but still feel restored and rested in the morning. ${ }^{18}$

Affiliations: Université de Montreal, Centre de Recherche, Institut Universitaire de Gériatrie de Montréal, Montréal, Que., Canada.

Competing interests: None declared.

DOI: 10.1503/jpn.140355

\section{References}

1. Billioti de Gage S, Moride Y, Ducruet T, et al. Benzodiazepine use and risk of Alzheimer's disease: case-control study. BMJ 2014;349:g5205.

2. American Geriatrics Society 2012 Beers Criteria Update Expert Panel. American Geriatrics Society updated Beers Criteria for potentially inappropriate medication use in older adults. J Am Geriatr Soc 2012;60:616-31. 
3. Tannenbaum C, Paquette A, Hilmer S, et al. A systematic review of amnestic and non-amnestic mild cognitive impairment induced by anticholinergic, antihistamine, GABAergic and opioid drugs. Drugs Aging 2012;29:639-58.

4. Woolcott JC, Richardson KJ, Wiens MO, et al. Meta-analysis of the impact of 9 medication classes on falls in elderly persons. Arch Intern Med 2009;169:1952-60.

5. Zint K, Haefeli WE, Glynn RJ, et al. Impact of drug interactions, dosage, and duration of therapy on the risk of hip fracture associated with benzodiazepine use in older adults. Pharmacoepidemiol Drug Saf 2010;19:1248-55.

6. Dassanayake T, Michie P, Carter G, et al. Effects of benzodiazepines, antidepressants and opioids on driving: a systematic review and meta-analysis of epidemiological and experimental evidence. Drug Saf 2011;34:125-56.

7. Hampton LM, Daubresse M, Chang HY, et al. Emergency department visits by adults for psychiatric medication adverse events. JAMA Psychiatry 2014;71:1006-14.
8. Weich S, Pearce HL, Croft P et al. Effect of anxiolytic and hypnotic drug prescriptions on mortality hazards: retrospective cohort study. BMJ 2014;348:g1996.

9. Martin P, Ahmed S, Tamblyn R, et al. A drug education tool developed for older adults changes knowledge, beliefs and risk perceptions about inappropriate benzodiazepine prescriptions in the elderly. Patient Educ Couns 2013;92:81-7.

10. Tannenbaum C, Martin P, Tamblyn R, et al. Reduction of inappropriate benzodiazepine prescriptions among older adults through direct patient education: the EMPOWER cluster randomized trial. JAMA Intern Med 2014;174:890-8.

11. Vicens C, Bejarano F, Sempere E, et al Comparative efficacy of two interventions to discontinue long-term benzodiazepine use: cluster randomised controlled trial in primary care. $\mathrm{Br}$ Psychiatry 2014;204:471-9.

12. Paquin AM, Zimmerman K, Rudolph JL. Risk versus risk: a review of benzodiazepine reduction in older adults. Expert Opin Drug Saf 2014;13:919-34.
13. Ashton $\mathrm{CH}$. Benzodiazepines: how they work and how to withdraw. 2002 Available: www. benzo.org.uk/manual/ (accessed 2014 Oct. 20).

14. Cook JM, Biyanova T, Masci C, et al. Older patient perspectives on long-term anxiolytic benzodiazepine use and discontinuation: a qualitative study. J Gen Intern Med 2007;22:1094-100.

15. Buysse DJ. Insomnia. JAMA 2013;309: 706-16.

16. Morin CM, Benca R. Chronic insomnia. Lancet 2012;379:1129-41.

17. Morin CM, Bastien C, Guay B, et al. Randomized clinical trial of supervised tapering and cognitive behavior therapy to facilitate benzodiazepine discontinuation in older adults with chronic insomnia. Am J Psychiatry 2004; 161:332-42.

18. Copinschi G, Caufriez A. Sleep and hormonal changes in aging. Endocrinol Metab Clin North Am 2013;42:371-89. 